\title{
Dietary Tyrosine/Phenylalanine Depletion Effects on Behavioral and Brain Signatures of Human Motivational Processing
}

\author{
James M Bjork*,', Steven J Grant ${ }^{1}$, Gang Chen ${ }^{2}$ and Daniel W Hommer ${ }^{*, 3}$ \\ 'Division of Clinical Neuroscience and Behavioral Research, National Institute on Drug Abuse, National Institutes of Health, Bethesda, MD, \\ USA; ${ }^{2}$ Scientific and Statistical Computing Core, National Institute of Mental Health, National Institutes of Health, Bethesda, MD, USA; \\ ${ }^{3}$ Laboratory of Clinical and Translational Studies, National Institute on Alcohol Abuse and Alcoholism, National Institutes of Health, Bethesda, \\ MD, USA
}

\begin{abstract}
Dopamine (DA) neurotransmission is critical for motivational processing. We assessed whether disruption of DA synthesis in healthy controls using an amino-acid beverage devoid of catecholamine precursors (tyrosine-phenylalanine depletion (TPD)) would blunt recruitment of the nucleus accumbens (NAcc) by rewards. Sixteen controls ingested each of a tyr/phe-depleting beverage (DEP) or a tyrl phe-balanced (BAL) control beverage in two laboratory visits. Five hours after consumption of each drink, subjects underwent functional magnetic resonance imaging while they viewed anticipatory cues to respond to a target to either win money or avoid losing money. TPD did not exert main effects on mood or on task behavior, but affected brain activation. In right NAcc, TPD blunted activation by anticipation of high rewards. In left NAcc, recruitment anticipating high rewards was modulated by individual differences in mood change across the DEP drink day, where subjects whose mood worsened following TPD (relative to within-day mood change under BAL conditions) also showed lower activation under DEP conditions relative to BAL conditions. Exploratory analysis indicated that TPD qualitatively blunted the voxel-wise spatial extent of suprathreshold activation by reward anticipation. Finally, loss outcomes activated anterior insula under DEP conditions but not under BAL conditions. These data indicate that: (I) dietary depletion of catacholamine precursors will blunt dopaminergic mesolimbic activity, and (2) in controls, synthetic pathways of this neurocircuitry maintain sufficient buffering capacity to resist an effect on motivated behavior. Additional studies are needed to determine if clinical populations would show similar resistance to behavioral effects of TPD.

Neuropsychopharmacology (2014) 39, 595-604; doi:I0.1038/npp.2013.232; published online 25 September 2013
\end{abstract}

Keywords: reward; dopamine; $\mathrm{fMRl}$; striatum; tyrosine; behavior

\section{INTRODUCTION}

Understanding the role of dopamine (DA) in human incentive-motivational behavior has considerable clinical importance. First, impaired DA functioning is thought to mediate anhedonia and motor symptoms in depression (Stein, 2008). Second, reward deficiency syndrome in addiction has been attributed to impaired DA function (Blum et al, 2000). A key region to interrogate is the ventral striatum (VS), including nucleus accumbens (NAcc) (Haber and Knutson, 2010; Schultz, 2007). Reward-related signaling involves phasic activity of dopaminergic neurons that project to the VS (Hernandez et al, 2007; Stuber et al,

*Correspondence: Dr JM Bjork, Division of Clinical Neuroscience and Behavioral Research, National Institute on Drug Abuse, National Institutes of Health, 600 I Executive Boulevard, Room 3163, Bethesda, MD 20892, USA, Tel: + I 30I 443 3209, Fax: + I 30| 443 68I4,

E-mail: jbjork@mail.nih.gov

Deceased.

Received 21 May 2013; revised 26 August 2013; accepted 27 August 2013; accepted article preview online 2 September 2013
2008). Blood oxygen level-dependent (BOLD) signal increases in VS have been frequently elicited by reward-predictive signals or reward deliveries during functional magnetic resonance imaging (fMRI), and this has been inferred as a marker of phasic dopaminergic activity (Knutson et al, 2001). Notably, individual differences in VS recruitment by reward anticipation (assessed with fMRI) during a monetary incentive delay (MID) task correlated with individual differences in displacement of ${ }^{11} \mathrm{C}$ raclopride in the VS by MID task rewards themselves (Schott et al, 2008), and with displacement of ${ }^{18} \mathrm{~F}$ fallypride by amphetamine (Buckholtz et al, 2010).

Inhibition of catecholamine synthesis with $\alpha$-methylpara-tyrosine blunted caudate activation during reward anticipation in the MID task (da Silva Alves et al, 2011). An alternative intervention, tyrosine-phenylalanine depletion (TPD), entails ingestion of an amino-acid beverage lacking catecholamine precursor L-tyrosine (Tyr) as well as L-phenylalanine (Phe), which is peripherally converted into Tyr by phenylalanine hydroxylase. Large neutral amino acids (LNAAs) compete with Tyr for CNS uptake 
(Oldendorf, 1973) and stimulate hepatic protein synthesis to incorporate extant Tyr into proteins (Harper et al, 1970). This markedly lowers concentrations of Tyr in the plasma (Moja et al, 1996; Palmour et al, 1998) as well as catecholamine metabolites in the CSF (Palmour et al, 1998) and striatum (Biggio et al, 1976) within hours. Perturbations of Tyr/Phe availability have altered responses to DA-mediated pharmacological or environmental challenges (reviewed in Milner and Wurtman, 1986; Tam and Roth, 1997). In humans, TPD blunted mood effects (thought to be DA-dependent) but not anorexic effects (thought to be NE-dependent; Silverstone, 1983) of amphetamine challenge (McTavish et al, 1999c). TPD also increased plasma prolactin (Harmer et al, 2001) by impairing dopaminergic lactotrophs of the tuberoinfundibular system.

Subjects performed the MID task after consuming a Tyr/ Phe-deficient amino-acid beverage (DEP), and also after consuming a balanced (BAL) amino-acid beverage containing proportional Tyr and Phe. We hypothesized that TPD would blunt VS activation by reward-predictive cues (Nagano-Saito et al, 2012). Because NAcc recruitment during reward anticipation has correlated with self-reported positive affect (Bjork et al, 2004; Knutson et al, 2001), we further hypothesized that blunted NAcc recruitment by rewards following TPD would be specific to those subjects who showed worsened mood. Finally, because mesolimbic DA neurons show phasic decreases in response to omissions of expected reward (Schultz, 2007), we hypothesized that occasional replacement of reward outcomes with a demand to repeat the trial would deactivate the NAcc (Bjork et al, 2008), but that this would be blunted following TPD owing to reduction in DA tone.

\section{MATERIALS AND METHODS}

\section{Subjects}

Procedures were approved by the Institutional Review Board of the National Institute on Alcohol Abuse and Alcoholism. Subjects $(n=16,10$ males; age 22-41, mean $29.2 \pm 5.2$ years) provided written informed consent and underwent physical examination and a structured clinical interview for DSM-IV. The exclusion criteria were as follows: history of an Axis I psychiatric disorder, lefthandedness, pregnancy, current or past use of psychotropic medications, chronic medical conditions, or history of significant head injury or neurological disorder. Illicit drug abstinence was confirmed with urinalysis.

\section{Experiment Schedule}

Each subject was tested in two sessions at least 1 week apart. Female subjects were tested during the follicular phase of the menstrual cycle. Each subject was instructed not to eat after 2200 hours before the test day. Subjects underwent baseline venipuncture at 0930 hours. At 0945 hours, subjects completed a baseline mood rating with the Profile of Mood States (POMS) (McNair et al, 1992), and then ingested one of the two amino-acid beverages (described below) at 1000 hours. At 1430 hours, subjects underwent postdrink venipuncture and POMS readministration, and then were scanned at 1500 hours to correspond with plasma nadirs of Tyr and Phe that occur 5-6 h after ingestion of the DEP beverage (Leyton et al, 2000; Moja et al, 1996). Subjects were scanned during three runs of the MID task, and then were administered a high-resolution structural scan for colocalization of functional images.

\section{Amino-Acid Administration}

Amino-acid mixtures were administered in a randomized, double-blind manner. On one visit, the subject ingested the Tyr/Phe-depleting beverage (DEP condition). On the other visit (order counterbalanced), the subject consumed a beverage containing balanced amounts of Tyr and Phe (BAL condition) (see Supplementary Methods). The amino acids were mixed with water and either chocolate or orange flavoring to a volume of $500 \mathrm{ml}$. Methionine was administered in capsules. The dosage for female subjects was reduced by $20 \%$ for all amino acids.

\section{MID Task}

Stimuli were back-projected on a screen at the foot of the scanner bed and viewed using a head coil mirror. In the MID task used here, some reward trial outcomes were replaced by a demand to repeat the trial (Bjork et al, 2008). Trials consisted of: cue presentation, target presentation, and either success-dependent feedback or notification of the requirement to repeat the trial (Figure 1). All task stimuli were separated by presentation of a fixation crosshair for a jittered, uniformly distributed interval of either 2,4 , or $6 \mathrm{~s}$.

First, a cue shape was presented for $250 \mathrm{~ms}$, which signaled what the subject would win for hitting a target: low

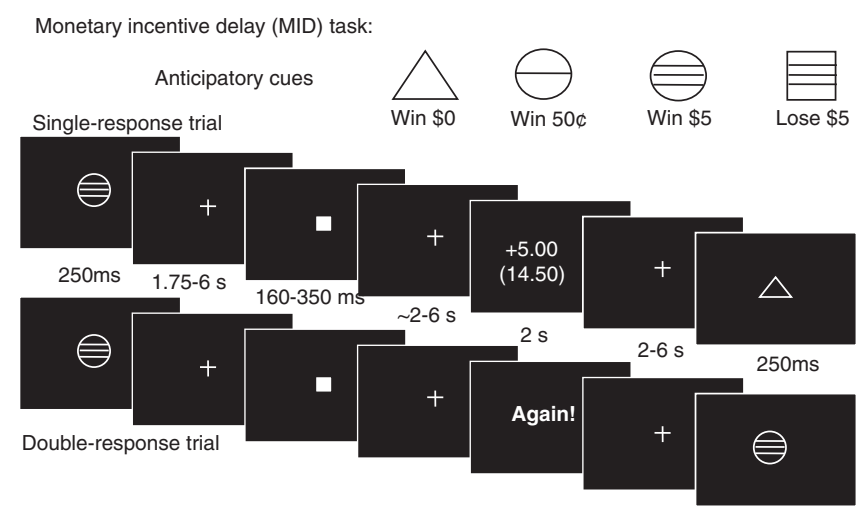

Figure I Monetary incentive delay (MID) task. In each trial, subjects were initially presented with one of four anticipatory cues followed by a fixation crosshair for $1.75-6 \mathrm{~s}$, and a target followed by a post-target fixation crosshair for $\sim 2-6 \mathrm{~s}$. Subjects were required to respond during target presentation ('hit') to either win money, avoid losing money, or for no consequence. In single-response trials, subjects next viewed notification of whether or not the target was hit, followed by a 2-6s intertrial interval with fixation crosshair. In double-response trials (one-third of each of win $\$ 0$, win $50 \phi$, and win $\$ 5$ magnitudes), trial outcome notifications were pseudorandomly replaced with the word 'Again!' This notified the subject that he or she must repeat the trial to obtain its outcome. The 'Again!' notification was followed by: a 2-6s fixation crosshair, re-presentation of the same incentive cue, jittered $(1.75-6 \mathrm{~s})$ postcue fixation, target presentation of the same duration as the trial's initial target, followed by $\sim 2-6 \mathrm{~s}$ fixation crosshair, and final outcome notification. In doubleresponse trials, hits on both targets were required for trial success. 
reward ( $50 \notin ; 27$ trials), high reward ( $\$ 5 ; 27$ trials), or no reward ( $\$ 0 ; 27$ trials). Second, the cue was replaced by a crosshair for a variable interval $(2,4$, or $6 \mathrm{~s})$. Third, a white target square was presented for $180-450 \mathrm{~ms}$. Subjects were instructed to respond to every target. To succeed, the subject was required to press a button on a button box while the target was on-screen. After a 2-6s delay following the target, the trial concluded with a $2 \mathrm{~s}$ presentation of either: (a) feedback of whether the subject hit the target (singleresponse trials; $n=18$ ) or (b) the word 'Again!', which notified the subject that the cue and target for the trial would be re-presented before an outcome would be delivered (double-response trials; $n=9$ ). In each doubleresponse trial, the same cue and target duration were repeated, and then were followed by feedback, where hits on both the first and second target were required to obtain reward. In loss-avoidance trials $(n=18)$, a striped square cue $(250 \mathrm{~ms})$ signaled the possibility of losing $\$ 5$ if the subject failed to hit the target. Loss-avoidance trials were single-response only.

Before scanning, subjects were shown an envelope containing the cash they could win, and were read an instruction script. Subjects were briefed that in some trials (double-response trials) they would be required to hit the target on two presentations to win. Later, during a practice session, reaction times (RTs) to targets were covertly measured, and the distribution of target presentation durations was rigged so that each participant would succeed on $\sim 67 \%$ of trials during the scan. Immediately after scanning, subjects rated on four-point scales how 'excited', 'happy', 'fearful', and 'unhappy' they felt when they saw each type of anticipatory cue or the 'Again!' demand.

\section{Behavior and Mood Analyses}

RTs were analyzed with repeated-measures analyses of variance, with mixture (two levels: BAL, DEP), incentive (four levels: non-incentive, win $50 \notin$, win $\$ 5$, and lose $\$ 5$ ), and time (three levels: scan runs 1-3) as nested withinsubject factors. Analysis of double-response trial types added target attempt (initial $v s$ repeated) as a withinsubject factor. Task hit rates were also analyzed with repeated-measures analyses of variance, with mixture and incentive amount as within-subject factors. Analysis of POMS used raw scores and was restricted to the total mood disturbance (TMD) composite score to reduce comparisons. TMD ratings were compared before and $5 \mathrm{~h}$ after ingestion of each of the DEP and BAL conditions, using a $2 \times 2$ ANOVA. Postscan affective ratings were also compared across incentive magnitudes and between the two mixture conditions using repeatedmeasures ANOVA.

\section{Plasma Amino-Acid Analysis}

Free plasma amino-acid concentrations were measured with the EZ:faast kit (Phenomenex, Torrance, CA) and analyzed as the concentration of tyrosine relative to the sum of concentrations of LNAAs administered in the drink (Tyr/LNAA ratio).

\section{FMRI Acquisition}

Imaging was performed using a $3 \mathrm{~T}$ MRI scanner (General Electric, Milwaukee, WI) with an eight-channel head coil. Functional scans were acquired using a $\mathrm{T} 2{ }^{*}$-sensitive echoplanar sequence with $\mathrm{TR}=2000 \mathrm{~ms}$, echo time $=30 \mathrm{~ms}$, and flip $=90^{\circ}$. We collected 24 4.0-mm-thick sagittal slices. In-plane resolution was $3.75 \times 3.75 \mathrm{~mm}^{2}$. Structural scans were acquired using a T1-weighted sequence for coregistration of functional data. Each subject's head was restrained with a deflateable head cushion.

\section{FMRI Analysis}

Preprocessing. Data sets were time-shifted to compensate for non-simultaneous slice acquisition, warped out into Talairach space as $3.75 \mathrm{~mm}$ isotropic voxels, corrected for head motion, and spatially smoothed to a uniform $6 \mathrm{~mm}$ full-width half-maximum. Time series were modeled with gamma variate hemodynamic responses time-locked to anticipatory cues, targets, presentation of 'Again!' commands, or outcome notifications. Activations were detected by five linear contrasts (hereafter 'contrasts'): (1) Anticipation of responding for all rewards $(50 \varnothing$ and \$5) $v s$ for no incentive (\$0). For this contrast, signal in single-response trials was pooled with the first cue of double-response trials. (2) Anticipation of responding to avoid losses (\$5) vs for no incentive (\$0). (3) Notification of successful $v s$ unsuccessful outcomes in single-response reward trials. (4) Notification of losses $v s$ avoided losses in the loss-avoidance trials. Finally, to approximate brain activation elicited by omission of expected reward, 'Again!' notifications were contrasted with notification of non-wins in single-response trials.

Volume-of-interest analysis of NAcc signal change. The primary data of this experiment were the patterns of reward-anticipatory signal change in the NAcc. Each subject's hemodynamic responses were: (1) trial-averaged, (2) modeled for low-frequency baseline drifts, and (3) passed through a mask in gray matter at the ventromesial intersection of caudate and putamen at Talairach $Y=10$. The effect estimates ( $\beta$-values) as measures of response (in percent signal change) to each of the task stimuli were analyzed using a combination of repeated-measures ANO$\mathrm{VA}$ and paired $t$-tests.

Groupwise and between-group statistical mapping. We also conducted a brain-wide search for task activations under each mixture condition, as well as the net difference in task activation (DEP conditions relative to BAL). Statistical maps were calculated in AFNI using 3dMEMA (http://afni.nimh.nih.gov/sscc/gangc/MEMA.html), with a linear mixed-effects multilevel model that incorporates both within- and cross-subject variability. The contrast between the two mixture conditions was analyzed with 3dMEMA. Group activations in each mixture condition were controlled by false discovery rate (FDR) correction. In the supplementary tables, these are reported at the maxima of activated voxel clusters. In the figures, FDR-surviving voxels are illuminated at a voxelwise significance threshold of $P<0.001$. 


\section{RESULTS}

\section{Plasma Amino-Acid Levels}

Blood samples were not available for four subjects. The BAL drink resulted in no significant change in the plasma-free Tyr/LNAA ratio (repeated-measures ANOVA $P=0.68$ ) across the day, whereas the DEP drink caused a substantial reduction in the Tyr/LNAA ratio $\mathrm{F}(1,11)=94.635$, $P<0.0001$ ) in every subject (mean values in Figure 2a), resulting in a significant mixture $\times$ time interaction effect $(\mathrm{F}(1,11)=23.803, P<0.0001)$.

\section{Mood Responses Under Different Mixture Conditions}

Subjects reported variable, but generally positive affect (Figure $2 \mathrm{~b}$ ). There were no main effects of either time (pre$v s$ postdrink) or of amino-acid mixture (BAL vs DEP) on TMD, nor was there a significant time $\times$ mixture interaction effect on TMD. Self-reported excitement $(\mathrm{F}(2,30)=135.870$, $P<0.000001)$ and happiness $(\mathrm{F}(2,30)=55.886, P<0.000001)$ evoked by reward-anticipatory cues increased with incentive magnitude $(\$ 0,50 \notin, \$ 5)$. Similarly, self-reported fearfulness $(\mathrm{F}(1,15)=198.818, P<0.000001)$ and unhappiness $(\mathrm{F}(1,15)=45.996, P<0.00001)$ evoked by loss-anticipatory cues significantly increased with magnitude $(\$ 0, \$ 5)$. In double-response trials, self-reported fearfulness $(\mathrm{F}(2,30)$ $=25.413, P<0.000001)$ and unhappiness $(\mathrm{F}(2,30)=6.530$, $P<0.01)$ upon viewing the 'Again!' demand to repeat the trial also increased with incentive magnitude $(\$ 0,50 c, \$ 5)$ of the trial. There were no significant main or interaction effects of amino-acid mixture, however, on MID task affective ratings.

\section{MID Task Behavior}

RT to the sole target of single-response trials (pooled with the initial target of double-response trials) showed main effects of incentive $(F(3,45)=16.422, P<0.00001)$ but no main effects of mixture or time (Figure 2d). Subjects responded more quickly to gain or to avoid losing money than for no incentive. A significant time $\times$ incentive interaction $(\mathrm{F}(3,45)=3.531, P<0.01)$ effect indicated that RT to incentivized targets became faster across task runs, but RT to non-incentive targets did not. Double-response trials also indicated a main effect of incentive on RT $(\mathrm{F}(2,30)=17.016, \quad P<0.0001)$, but no main effect of response attempt on $\mathrm{RT}$, nor any mixture $\times$ attempt or incentive $\times$ attempt interaction effects. Because target durations did not differ by incentive type, hit rates were accordingly lower for non-incentive targets relative to incentivized targets (main effect of incentive $(\mathrm{F}(3,48)=$ 44.973, $P<0.000001$ ). There were no significant main or interaction effects of mixture on hit rates.

\section{Task-Elicited Signal Change in NAcc (VOI Analysis)}

Responses to anticipatory cues increased with incentive amount under both mixture conditions (main effect of magnitude $\mathrm{F}(2,30)=19.702, P<0.00001)$, where non-incentive

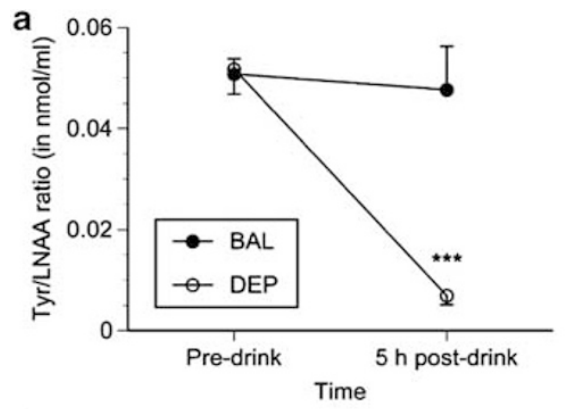

d

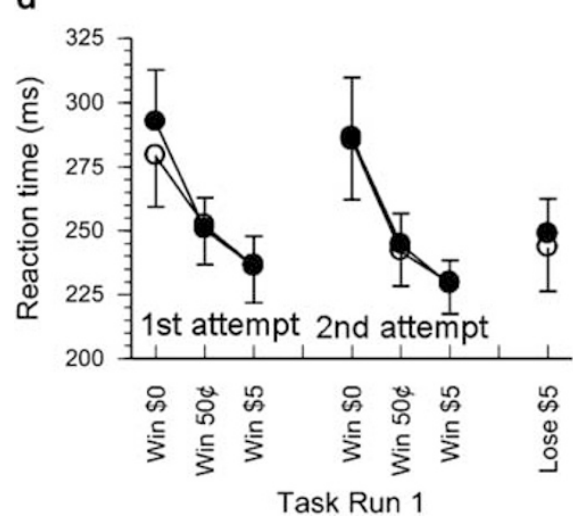

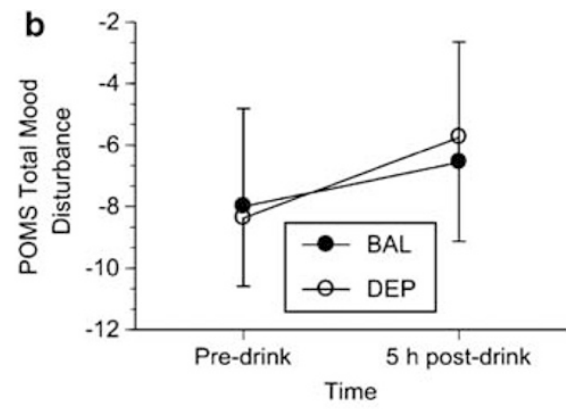
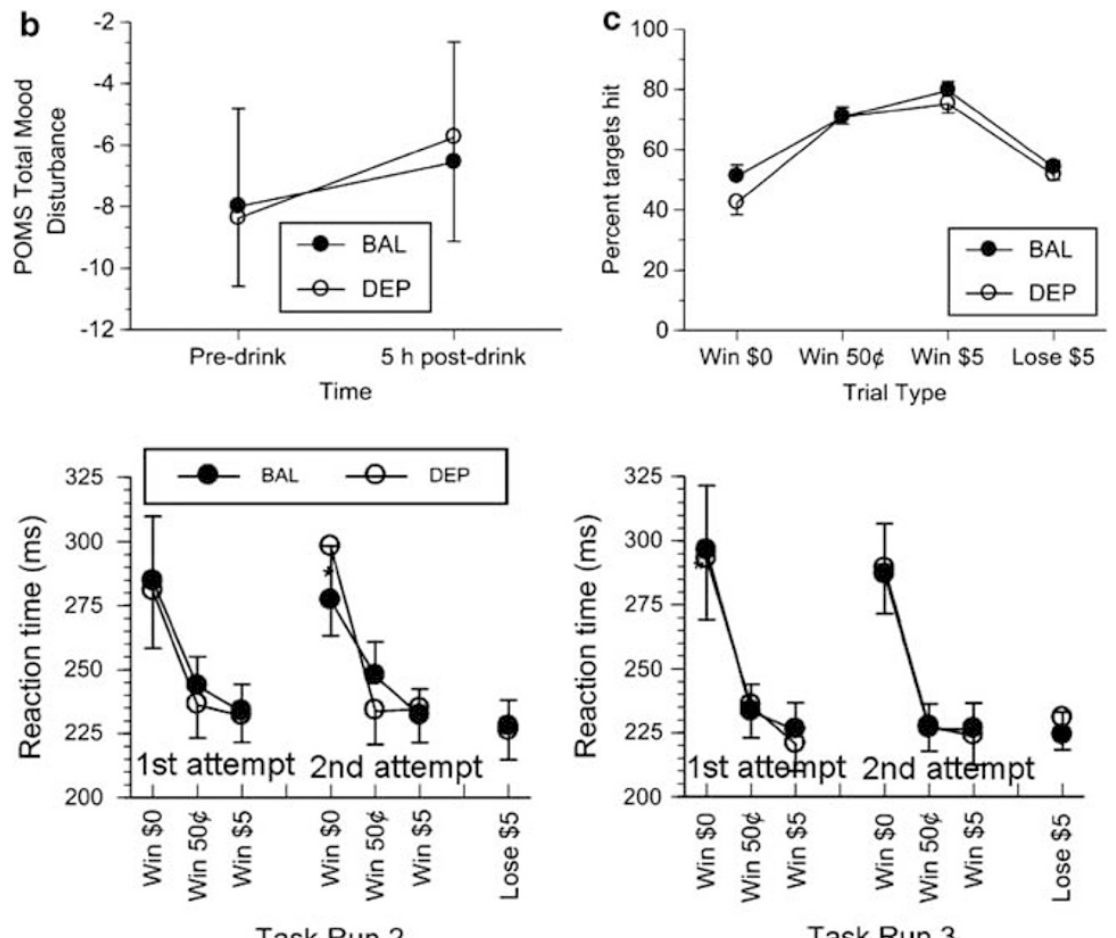

Task Run 2

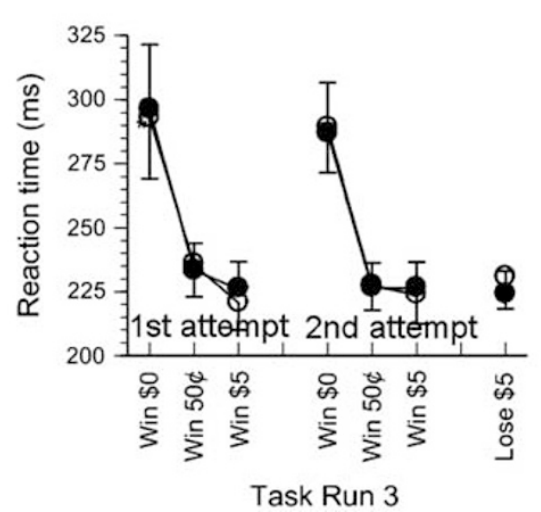

Figure 2 Effects of tyrosine-phenylalanine depletion (TPD) on free plasma amino-acid concentrations, mood, and task behavior. Acute TPD (tyr/phedepleting beverage (DEP) beverage), but not the balanced-concentration (BAL) beverage resulted in a substantial reduction in the ratio of catecholamine precursor Tyr relative to competing large neutral amino acids (LNAA) in the plasma obtained $5 \mathrm{~h}$ after amino-acid beverage consumption (a). TPD had no effect, however, on Total Mood Disturbance as self-reported on the Profile of Mood States (POMS) (b). Target hit rates on the monetary incentive delay (MID) task (excluding second responses of double-response trials) varied as a function of the incentive at stake in the trial (c), where these differences in hit rates resulted from significant differences in reaction times to targets under different incentive conditions (d). ***** denotes mixture $\times$ time interaction effect at $P<0.000$ l. 


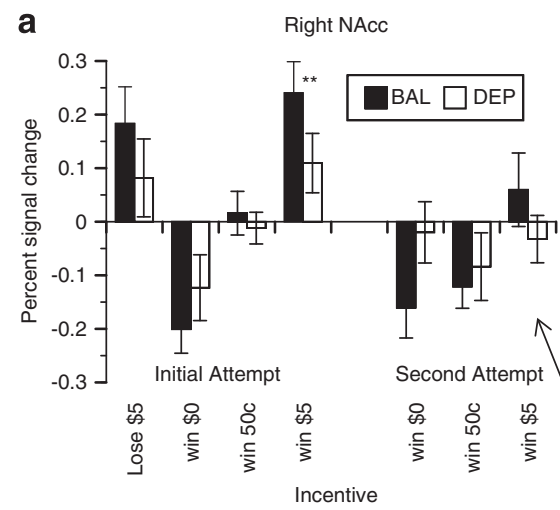

b Left NAcC
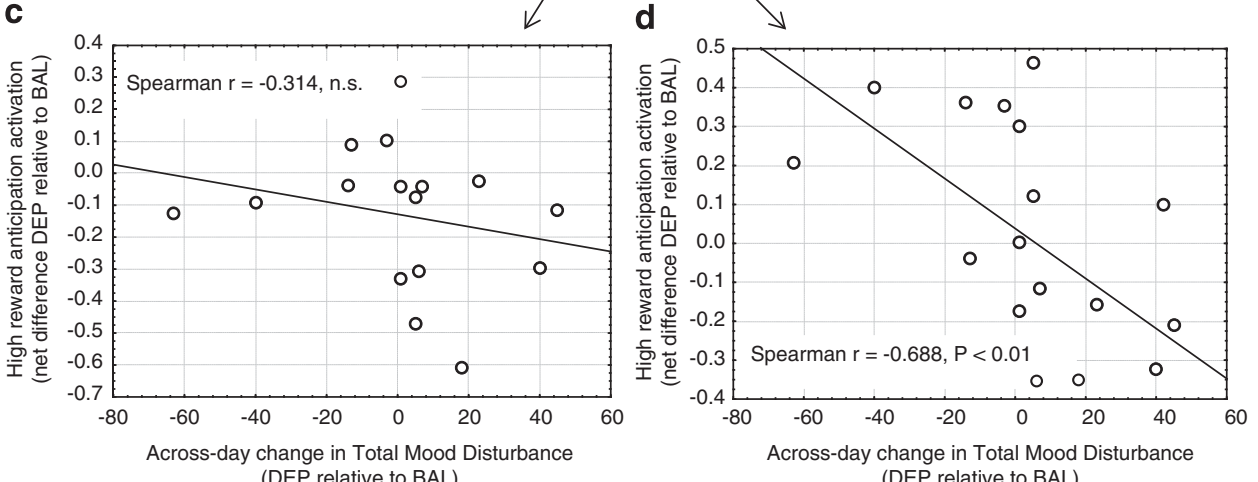

(DEP relative to $B A L$ )

(DEP relative to $B A L)$

Figure 3 Anticipation cue-elicited signal change in the nucleus accumbens (NAcc) volume of interest (VOI). In a VOI drawn in the NAcc bilaterally (Talairach $\pm 10+10-4$; inset) modeled peak anticipatory blood oxygen level-dependent (BOLD) signal change reflected the prospective incentive value of hitting the target in both right (a) and left (b) Nacc. Notably, a significant attempt $\times$ magnitude interaction effect indicated that this magnitude sensitivity of the NAcc responses was specific to initial/only attempt to hit the target, but not in second (repeat) responses to hit in the double-response trials. Across both sides of the NAcc, a significant mixture $\times$ magnitude interaction effect suggested reduced reward-magnitude sensitivity under tyr/phe-depleting beverage (DEP) conditions compared with balanced (BAL) conditions. The net change (increase) in total mood disturbance across the day following tyrosine/phenylalanine depletion (TPD) relative to change under BAL conditions correlated with the net blunting of activation by high reward anticipation under DEP conditions relative to BAL conditions in left NAcc (d) but not right NAcc (c). * denotes mixture-wise $P<0.10, * * P<0.05$.

cues deactivated the VS, low-reward cues had minimal effect, and cues for high rewards and for losses activated VS (Figure 3). There were no main effects of side (left $v s$ right), attempt (first or second), or amino-acid mixture on anticipatory NAcc activation. There was a significant magnitude $\times$ attempt interaction effect $(\mathrm{F}(2,30)=12.835$, $P<0.0001$ ), however, where the effect of magnitude was specific to the initial cue presentation of double-response trials. Notably, a significant mixture $\times$ magnitude interaction effect $(\mathrm{F}(2,30)=4.352, P<0.05)$ indicated that magnitude effects on anticipatory activation were more pronounced under BAL conditions, whereas under DEP conditions, peak signal change in NAcc was more similar across trial incentives $(\$ 0,50 \notin, \$ 5)$. Post hoc t-tests indicated a significant reduction in anticipation of responding for high rewards in right NAcc (first cue) and a trend for a reduction in left NAcc (second cue) under DEP conditions relative to BAL conditions. Activation by anticipation of potential losses indicated no main or interaction effects of mixture or side. There were no main effects of side, mixture, magnitude, or outcome (hit $v s$ miss) on NAcc activation by trial notifications. There were no significant main or interaction effects of side, mixture, or magnitude in hemodyamic responses to the 'Again!' demand to repeat a trial in double-response trials.

Correlation between TPD effects on brain activation by rewards and TPD effects on mood. We wished to determine whether blunted NAcc responses to high reward cues under DEP conditions were specific to subjects who reported worsening mood following TPD. To limit comparisons, we only analyzed anticipatory signal change elicited by initial/only high reward cues. We calculated the net difference in modeled peak BOLD signal response in NAcc elicited by high reward anticipation under DEP conditions minus the response under BAL conditions. Second, we calculated a POMS-TMD net change score as: $\left(\mathrm{TMD}_{\text {post-DEP }}-\right.$ $\left.\mathrm{TMD}_{\text {pre-DEP }}\right)-\left(\mathrm{TMD}_{\text {post-BAL }}-\mathrm{TMD}_{\text {pre-BAL }}\right)$. Across subjects, net worsening of mood following the DEP drink (relative to mood change following the BAL drink) correlated with lower NAcc reward-anticipatory activation under DEP conditions relative to BAL in left NAcc (Spearman's $r=-0.688, P<0.01$ ) (Figure 3). 


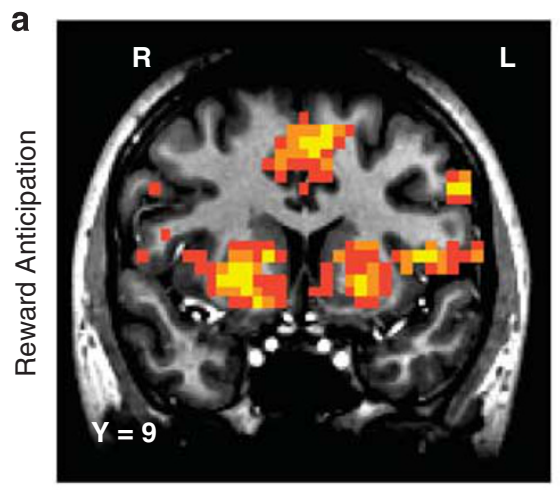

Balanced Drink (BAL)

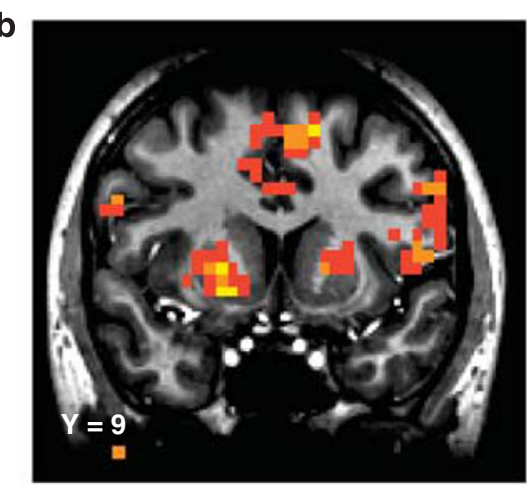

Depletion Drink (DEP)

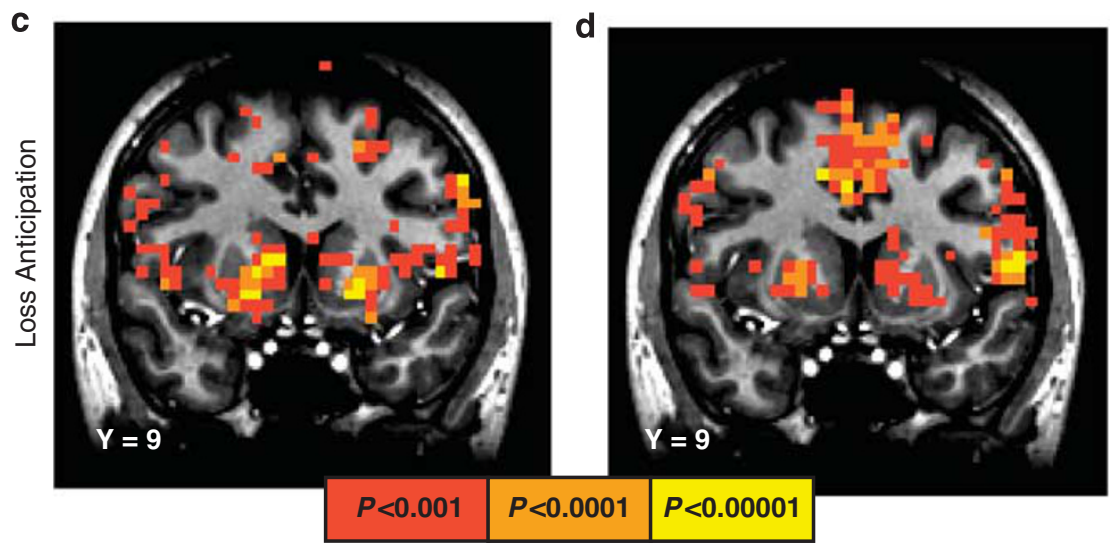

Figure 4 Statistical maps of activation by anticipation of potential rewards and losses. Anticipation of responding for rewards $(50 \phi$, $\$ 5)$ contrasted with anticipation of responding for no incentive activated bilateral ventral striatum under balanced (BAL) (a) and tyr/phe-depleting beverage (DEP) (b) amino-acid conditions. Anticipation of responding to avoid losses $(\$ 5)$ contrasted with anticipation of responding for no incentive also activated bilateral dorsal striatum in both BAL (c) and DEP (d) conditions. There were no voxel-wise amino-acid mixture differences in anticipatory activation for either potential rewards or losses. Statistical maps are right-left reversed per radiological convention, and anatomical underlays are derived from a $\mathrm{TI}$-wieghted scan of a representative subject at the planar Talairach coordinate shown. Color overlays depict uncorrected voxel-wise P statistics, where illuminated voxels or voxel clusters survive false discovery rate correction.

\section{Task Contrast Activations in Brain-Wide Search}

Reward vs non-incentive anticipation. Under BAL conditions, anticipation of responding for reward (50 $\varnothing$ and $\$ 5$ magnitudes combined) $v s$ responding for no incentive activated a large bilateral cluster of voxels in the VS, with activation extending into anterior insula (Figure 4 and Supplementary Table 1). Additional activations included ventromesial frontal cortex (vmFC), posterior mesofrontal cortex, and several portions of occipital cortex. Under DEP conditions, these same regions were activated (with the exception of vmFC), with a reduced spatial extent of suprathreshold activations. There were no activation differences between mixtures, after correction for multiple comparisons.

Loss vs non-incentive anticipation. Under BAL conditions, anticipation of responding to avoid a $\$ 5$ loss $v s$ responding for no incentive also activated a large bilateral cluster of voxels in the VS, with activation extending into anterior insula (Figure 4 and Supplementary Table 2). Additional activations by prospective losses also included vmFC, posterior mesofrontal cortex, and several portions of occipital cortex. Under DEP conditions, these same regions were activated (with the exception of vmFC), with a reduced spatial extent of suprathreshold activations. As with rewards, there were no direct mixture differences in lossanticipatory activation.

Gain vs nongain outcomes. This contrast did not yield activations that survived FDR correction in either the DEP or BAL conditions. Subthreshold (uncorrected voxelwise $P<0.001$ ) activations in vmFC by gain notifications were present under both mixture conditions (Figure $5 \mathrm{a}$ and $\mathrm{b}$ and Supplementary Table 3), with an additional activation of right NAcc by gains under DEP condition only. A direct mixture-difference map revealed no contrast activation differences as a function of amino-acid manipulation.

Loss vs avoided-loss outcomes. This contrast did not yield any activations under BAL conditions. Under DEP conditions, however, loss notifications elicited activation in the thalamus, left anterior insula, subgenual anterior cingulate cortex, and posterior mesofrontal cortex (Figure $5 \mathrm{c}$ and $\mathrm{d}$ and Supplementary Table 3).

'Again!' vs non-reward outcomes in reward trials. 'Again!' demands activated right anterior insula, bilateral inferior frontal lobe, and bilateral inferior occipital lobe under both DEP and BAL conditions (Figure $5 e$ and $f$ and 

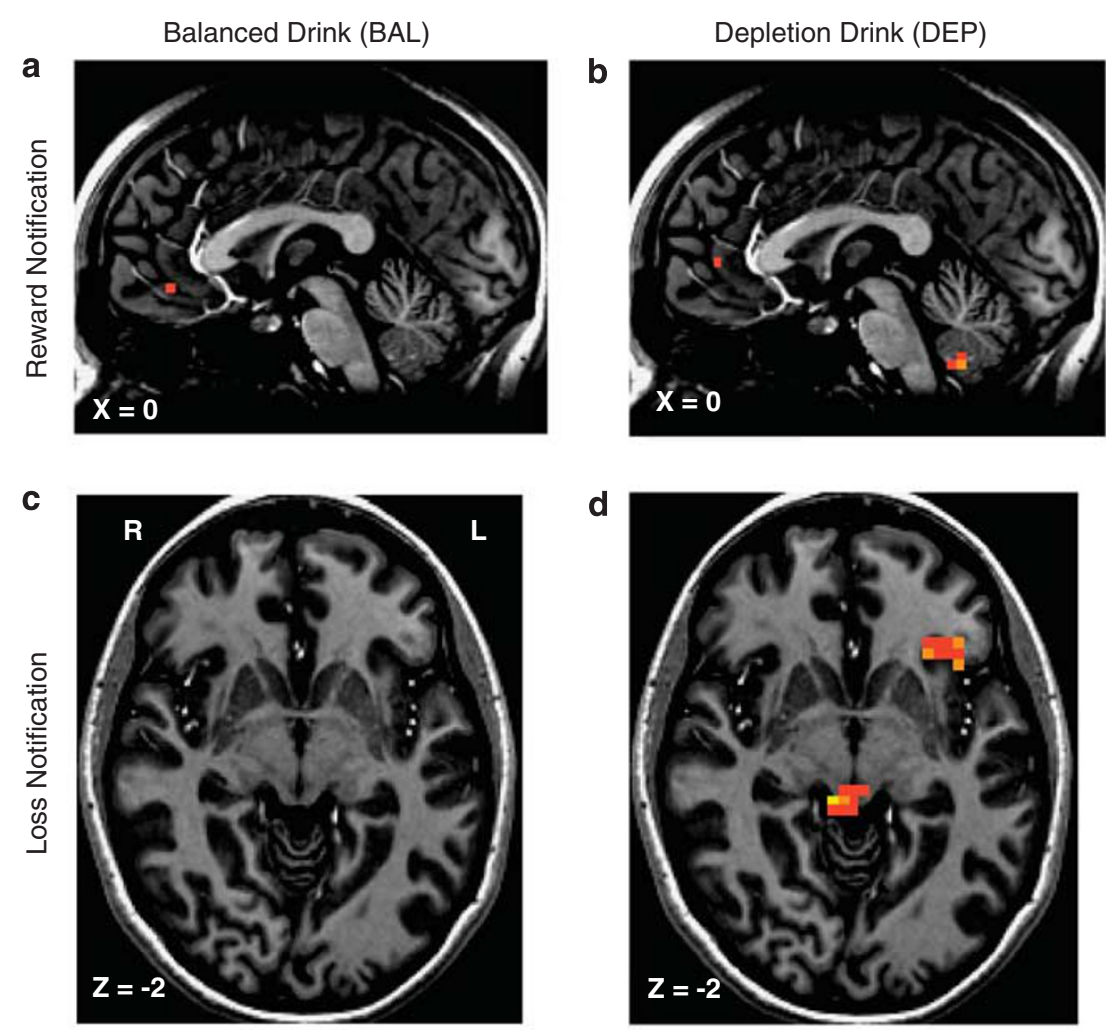

d
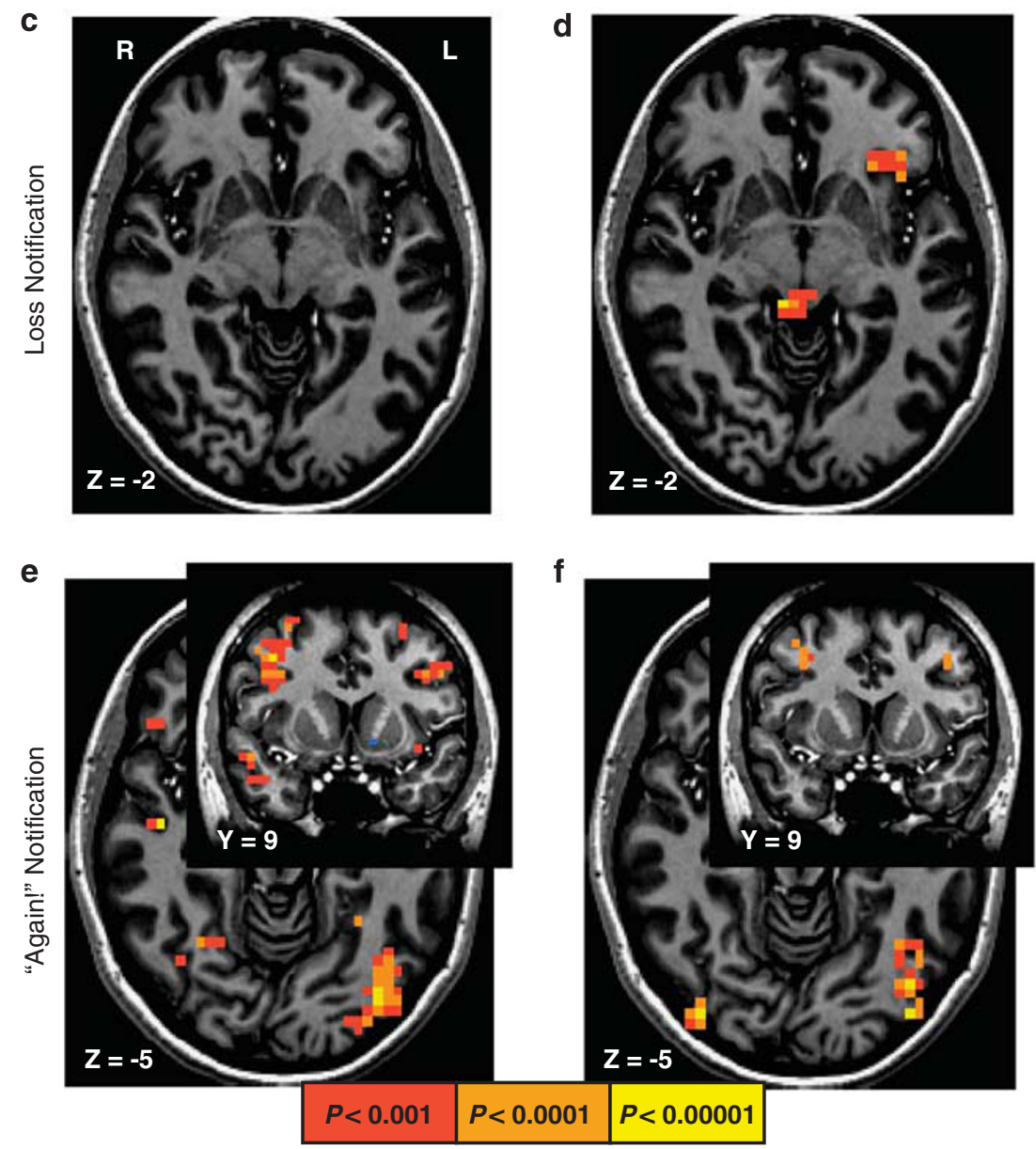

Figure 5 Statistical maps of activation by notification of rewards, by demands to repeat trial, and by notification of losses. Notification of rewards ( $50 \notin$, $\$ 5)$ contrasted with notification of failure to win reward activated a focal region of vmFC under both balanced (BAL) (a) and tyr/phe-depleting beverage (DEP) (b) conditions. Notification of losses (\$5) contrasted with notification of avoided losses did not activate any region above threshold under BAL conditions (c), but activated left anterior insula in under DEP conditions (d). Replacement of outcome notification with the demand to repeat the trial effort ('Again!') in reward trials $(50 \phi, \$ 5)$ contrasted with notification of non-wins in single-response reward trials activated inferior occipital and middle frontal cortex under both BAL (e) and DEP (f) conditions, with an additional suprathreshold deactivation of left ventral striatum (VS) under BAL conditions. vmFC, ventromesial frontal cortex.

Supplementary Table 4). Under BAL conditions (only), this contrast resulted in additional activations of precentral gyrus and left inferior parietal lobule, as well as a deactivation of left NAcc.

\section{DISCUSSION}

As we hypothesized, the administration of a large bolus of amino acids devoid of catecholamine precursors blunted brain activation by reward-predictive cues in the NAcc, a structure extensively implicated in the energizing of instrumental behavior (Haber and Knutson, 2010), presumably by reducing DA available for release. Notification of losses activated anterior insula under DEP conditions, but not under BAL conditions. Although there were no significant effects of TPD on task activation after brain-wide correction for multiple comparisons, we note that that the spatial extent of most task activations tended to be reduced under DEP conditions in the striatum and other regions with mesolimbic dopaminergic innervation. 
As we suspected, relatively reduced NAcc recruitment by reward anticipation under DEP conditions was specific to subjects who reported worsened mood following administration of the DEP beverage relative to the BAL beverage. Previously, blunted VS responses (Stoy et al, 2012) to reward cues and slower RTs (Hasler et al, 2009) to MID task targets have been found in clinically depressed subjects, with no activation differences in remitted (Dichter et al, 2012) or medicated (Stoy et al, 2012) depressed subjects. Of interest for future experiments would be collecting brain signal following TPD in subjects with histories of depression, anhedonia, or other affective disorder.

Our secondary hypothesis, that TPD would blunt VS deactivation by expected reward omission, was mildly supported. The contrast between 'Again!' notifications vs notification of failures to win in single-response trials elicited a significant focal left NAcc deactivation under BAL conditions, but not under DEP conditions. However, there were no direct significant mixture differences on VS deactivation by 'Again!' notifications. Although subject success on the large majority $(>70 \%)$ of rewarded trials likely created some expectation of wins, we did not collect information on subject expectancies.

Notification of gains activated vmFC under both mixture conditions, and also mildly activated right NAcc in the DEP condition. Mesolimbic DA signaling has been elicited by both learned reward-predictive signals as well as by receipt of unexpected rewards (Schultz, 2007). As cue-reward associations are MID task are already trained before the scan, and because reward outcomes are highly probable (here $\sim 75 \%$ ), we suspect that phasic striatal DA activity elicited by the MID task is most robust (and by extension vulnerable to TPD effects) during reward anticipation.

Notification of losses activated left anterior insula and subgenual anterior cingulate cortex under DEP (but not $\mathrm{BAL}$ ) conditions. Taken together with the presence of VS activation by gain notification under DEP (but not BAL) conditions, it is tempting to speculate that TPD may have increased limbic sensitivity to acute valenced instrumental outcomes through some other mechanism, to essentially mimic the responses of groups (eg, alcoholics) ostensibly characterized by impaired monoaminergic functioning. In the previous experiment with this MID task (Bjork et al, 2008), both left anterior insula activation by losses and VS activation by gains were greater in alcoholics. As we did not probe affective responses to gain and loss notifications, this question will require additional studies, where mood could be probed during the task.

RTs were essentially identical between the two mixture conditions. These behavioral data reflect previous findings in healthy controls, where TPD did not markedly affect RT in a simple signal-detection task (Nagano-Saito et al, 2012), and suggest that brain signal effects of TPD did not arise from sedation. Similar RT coupled with titration of target durations fostered consistent win rates across subjects and between mixtures. Therefore, NAcc activation differences between mixtures did not arise from differential framing of gains or losses against accumulation of winnings. Rather, mixture differences represented an absolute change in processing of the expected values of the specific trial magnitudes and outcomes.
Subjects generally reported positive mood, and TPD exerted no main effects on mood or on affective reactions to the anticipatory cues. The lack of a main effect of TPD on POMS-TMD ratings or MID task cue affective ratings also reflects negative findings in the literature when TPD is administered to normal controls (eg, Lythe et al, 2005; Coupland et al, 2001; Nagano-Saito et al, 2012) and even in subjects with histories of depression who were in remission at the time of testing (McTavish et al, 2004, 2005). There were large individual differences in absolute TMD ratings on each study day, however, and in net difference scores in POMS-TMD between mixture conditions. This also reflects previous findings of large individual differences in effects of TPD on mood (eg, Harmer et al, 2001).

In fMRI studies where brain activation differences are found in the absence of behavioral differences, activations are unconfounded by groupwise differences in success or frustration. Yet, a critical interpretive question arises: What is the functional meaning of the brain changes and the perturbation (or individual differences) that caused them? We contend that detection of covert markers (only) of brain alteration is meaningful (Wilkinson and Halligan, 2004). First, although controls showed a resistance to behavioral and mood effects of TPD, vulnerable populations might not. The literature is replete with examples of brain differences in response to a cognitive task in clinical populations, where the pathophysiological process had not yet advanced to the point of a behavioral decrement (eg, Ernst et al, 2009). Therefore, differences in brain signal (only) could provide an advanced signal of compromised processing. Second, that behavior and affect regarding the incentive cues remained intact despite the fact that some blunting of mesolimbic metabolic activity reveals a resistance of at least some elements of catecholaminergic neurocircuitry to acute precursor restriction. This indicates that synthetic pathways feature significant buffering capacity to maintain motivated behavior in the face of temporary changes in dietary resources. Finally, self-report measures of mood are subjective or insensitive, and can be prone to a variety of biases or other individual differences in self-awareness. In contrast, brain markers of incentive processing might represent a unbiased signal of valuation (Knutson et al, 2009).

These findings should be interpreted with some caveats. First, our sample size, while respectable for a repeatedmeasures pharmacological fMRI experiment, precluded examination of potential laterality differences in striatal activation, sex differences in mixture effects, as well as correlations between magnitude of plasma amino-acid level alterations and beverage effects on task activations or mood. Second, acute TPD is an imprecise intervention. The conventional BAL beverage was not a true 'placebo' and may have appreciably affected other transmitter systems. Second, although scans were scheduled at the nadir of plasma TyrPhe/LNAA ratio, when plasma prolactin concentrations (as a peripheral marker of reduced central DA function) previously elicited by this mixture were at their peak (Harmer et al, 2001), it is possible that the nadir of restricted central catecholamine synthesis occurred later (Palmour et al, 1998). Moreover, TPD decreases norepinephrine (NE) synthesis as well as DA synthesis (eg, Palmour et al, 1998). Therefore, activation changes cannot be definitively attributed to changes in DA synthesis. Other 
evidence, however, suggests that TPD disproportionately affects the DA system. McTavish et al (2001) demonstrated that TPD blunted locomotor responses to amphetamine, with disproportionate reductions in amphetamine-induced DA release in the striatum (McTavish et al, 1999b). In contrast, they reported a more modest effect of TPD on amphetamine-induced (McTavish et al, 1999b) and idazoxan-induced (McTavish et al, 1999a) NE metabolite concentrations.

In addition, both amino-acid mixtures depleted Lhistidine (His). His competes with other LNAAs for transit into the brain, where it is transformed into histamine, such that His restriction holds the potential for an antihistaminelike sedative effect. In a degraded-stimulus signal-detection task, however, His depletion only mildly affected some taskelicited EEG signatures, but did not significantly affect RTs or accuracy of signal detection (van Ruitenbeek et al, 2009). Similarly, RTs in this experiment were substantially similar to those of controls in the previous study with this MID variant (Bjork et al, 2008) that featured no pharmacological manipulation. However, it cannot be ruled out that some effects may have resulted from reduced histamine signaling (on both mixture days).

In conclusion, in healthy adults, TPD mildly reduced the overall dynamic response of the mesolimbic DA system to incentives, where this temporary disruption of catecholamine synthesis was not sufficient to cause a functional change in the incentive-driven behavior. We interpret this as evidence of the resilience of the brain's catecholaminergic pathways to dietary alterations. Future experiments could examine extended effects of DA precursor restriction, or could apply acute TPD to at-risk populations.

\section{FUNDING AND DISCLOSURE}

This research was sponsored by intramural research funds of the National Institute on Alcohol Abuse and Alcoholism. None of the authors has any conflict or competing interest regarding this research study and its findings, financial, or otherwise.

\section{ACKNOWLEDGEMENTS}

This paper is dedicated to the memory of Dr Daniel W Hommer. We thank Ashley R Smith for her dutiful subject recruitment and data collection, and Erick Singley for the plasma amino-acid analysis.

\section{REFERENCES}

Biggio G, Porceddu ML, Gessa GL (1976). Decrease of homovanillic, dihydroxyphenylacetic acid and cyclic-adenosine- $3^{\prime}$, $5^{\prime}$-monophosphate content in the rat caudate nucleus induced by the acute administration of an aminoacid mixture lacking tyrosine and phenylalanine. J Neurochem 26: 1253-1255.

Bjork JM, Knutson B, Fong GW, Caggiano DM, Bennett SM, Hommer DW (2004). Incentive-elicited brain activation in adolescents: similarities and differences from young adults. J Neurosci 24: 1793-1802.

Bjork JM, Smith AR, Hommer DW (2008). Striatal sensitivity to reward deliveries and omissions in substance dependent patients. NeuroImage 42: 1609-1621.
Blum K, Braverman ER, Holder JM, Lubar JF, Monastra VJ, Miller D et al (2000). Reward deficiency syndrome: a biogenetic model for the diagnosis and treatment of impulsive, addictive, and compulsive behaviors. J Psychoactive Drugs 32(Suppl i-iv): $1-112$.

Buckholtz JW, Treadway MT, Cowan RL, Woodward ND, Benning $\mathrm{SD}$, Li R et al (2010). Mesolimbic dopamine reward system hypersensitivity in individuals with psychopathic traits. Nat Neurosci 13: 419-421.

Coupland N, Zedkova L, Sanghera G, Leyton M, Le Melledo JM (2001). Response to pentagastrin after acute phenylalanine and tyrosine depletion in healthy men: a pilot study. J Psychiatry Neurosci 26: 247-251.

da Silva Alves F, Schmitz N, Figee M, Abeling N, Hasler G, van der Meer J et al (2011). Dopaminergic modulation of the human reward system: a placebo-controlled dopamine depletion fMRI study. J Psychopharmacol 25: 538-549.

Dichter GS, Kozink RV, McClernon FJ, Smoski MJ (2012). Remitted major depression is characterized by reward network hyperactivation during reward anticipation and hypoactivation during reward outcomes. J Affect Disord 136: 1126-1134.

Ernst T, Yakupov R, Nakama H, Crocket G, Cole M, Watters M et al (2009). Declined neural efficiency in cognitively stable human immunodeficiency virus patients. Ann Neurol 65: 316-325.

Haber SN, Knutson B (2010). The reward circuit: linking primate anatomy and human imaging. Neuropsychopharmacology 35: 4-26.

Harmer CJ, McTavish SF, Clark L, Goodwin GM, Cowen PJ (2001). Tyrosine depletion attenuates dopamine function in healthy volunteers. Psychopharmacology (Berl) 154: 105-111.

Harper AE, Benevenga NJ, Wohlhueter RM (1970). Effects of ingestion of disproportionate amounts of amino acids. Physiol Rev 50: 428-558.

Hasler G, Luckenbaugh DA, Snow J, Meyers N, Waldeck T, Geraci $M$ et al (2009). Reward processing after catecholamine depletion in unmedicated, remitted subjects with major depressive disorder. Biol Psychiatry 66: 201-205.

Hernandez G, Haines E, Rajabi H, Stewart J, Arvanitogiannis A, Shizgal P (2007). Predictable and unpredictable rewards produce similar changes in dopamine tone. Behav Neurosci 121: 887-895.

Knutson B, Adams CM, Fong GW, Hommer D (2001). Anticipation of increasing monetary reward selectively recruits nucleus accumbens. J Neurosci 21: RC159.

Knutson B, Delgado MR, Phillips PEM (2009). Representation of subjective value in the striatum. In: Glimcher PW, Camerer CF, Fehr E, Poldrack RA (eds). Neuroeconomics: Decision-Making and the Brain. Elsevier: New York, NY, pp 389-406.

Leyton M, Young SN, Blier P, Baker GB, Pihl RO, Benkelfat C (2000). Acute tyrosine depletion and alcohol ingestion in healthy women. Alcohol Clin Exp Res 24: 459-464.

Lythe KE, Anderson IM, Deakin JF, Elliott R, Strickland PL (2005). Lack of behavioural effects after acute tyrosine depletion in healthy volunteers. J Psychopharmacol 19: 5-11.

McNair DM, Lorr M, Droppleman LF (1992). Profile of Mood States. Educational and Industrial Testing ServiceSan Diego, CA.

McTavish SF, Callado L, Cowen PJ, Sharp T (1999a). Comparison of the effects of alpha-methyl-p-tyrosine and a tyrosine- free amino acid load on extracellular noradrenaline in the rat hippocampus in vivo. J Psychopharmacol 13: 379-384.

McTavish SF, Cowen PJ, Sharp T (1999b). Effect of a tyrosine-free amino acid mixture on regional brain catecholamine synthesis and release. Psychopharmacology (Berl) 141: 182-188.

McTavish SF, Mannie ZN, Cowen PJ (2004). Tyrosine depletion does not cause depressive relapse in antidepressant-treated patients. Psychopharmacology (Berl) 175: 124-126.

McTavish SF, Mannie ZN, Harmer CJ, Cowen PJ (2005). Lack of effect of tyrosine depletion on mood in recovered depressed women. Neuropsychopharmacology 30: 786-791. 
McTavish SF, McPherson MH, Sharp T, Cowen PJ (1999c). Attenuation of some subjective effects of amphetamine following tyrosine depletion. J Psychopharmacol 13: 144-147.

McTavish SF, Raumann B, Cowen PJ, Sharp T (2001). Tyrosine depletion attenuates the behavioural stimulant effects of amphetamine and cocaine in rats. Eur J Pharmacol 424: 115-119.

Milner JD, Wurtman RJ (1986). Catecholamine synthesis: physiological coupling to precursor supply. Biochem Pharmacol 35: 875-881.

Moja EA, Lucini V, Benedetti F, Lucca A (1996). Decrease in plasma phenylalanine and tyrosine after phenylalanine-tyrosine free amino acid solutions in man. Life Sci 58: 2389-2395.

Nagano-Saito A, Cisek P, Perna AS, Shirdel FZ, Benkelfat C, Leyton $\mathrm{M}$ et al (2012). From anticipation to action, the role of dopamine in perceptual decision making: an fMRI-tyrosine depletion study. J Neurophysiol 108: 501-512.

Oldendorf WH (1973). Stereospecificity of blood-brain barrier permeability to amino acids. Am J Physiol 224: 967-969.

Palmour RM, Ervin FR, Baker GB, Young SN (1998). An amino acid mixture deficient in phenylalanine and tyrosine reduces cerebrospinal fluid catecholamine metabolites and alcohol consumption in vervet monkeys. Psychopharmacology (Berl) 136: 1-7.

Schott BH, Minuzzi L, Krebs RM, Elmenhorst D, Lang M, Winz OH et al (2008). Mesolimbic functional magnetic resonance imaging activations during reward anticipation correlate with reward-related ventral striatal dopamine release. J Neurosci 28: 14311-14319.
Schultz W (2007). Behavioral dopamine signals. Trends Neurosci 30: $203-210$.

Silverstone T (1983). The clinical pharmacology of appetite-its relevance to psychiatry. Psychol Med 13: 251-253.

Stein DJ (2008). Depression, anhedonia, and psychomotor symptoms: the role of dopaminergic neurocircuitry. CNS Spectr 13: 561-565.

Stoy M, Schlagenhauf F, Sterzer P, Bermpohl F, Hagele C, Suchotzki K et al (2012). Hyporeactivity of ventral striatum towards incentive stimuli in unmedicated depressed patients normalizes after treatment with escitalopram. J Psychopharmacol 26: $677-688$.

Stuber GD, Klanker M, de Ridder B, Bowers MS, Joosten RN, Feenstra MG et al (2008). Reward-predictive cues enhance excitatory synaptic strength onto midbrain dopamine neurons. Science 321: 1690-1692.

Tam SY, Roth RH (1997). Mesoprefrontal dopaminergic neurons: can tyrosine availability influence their functions? Biochem Pharmacol 53: 441-453.

van Ruitenbeek P, Sambeth A, Vermeeren A, Young SN, Riedel WJ (2009). Effects of L-histidine depletion and L-tyrosine/L-phenylalanine depletion on sensory and motor processes in healthy volunteers. Br J Pharmacol 157: 92-103.

Wilkinson D, Halligan P (2004). The relevance of behavioural measures for functional-imaging studies of cognition. Nat Rev Neurosci 5: 67-73.

Supplementary Information accompanies the paper on the Neuropsychopharmacology website (http://www.nature.com/npp) 